\title{
Therapeutic fibreoptic bronchoscopy in intensive care
}

\author{
J S MILLEDGE
}

British Medical fournal, 1976, 2, 1427-1429

quite traumatic and distressing to the conscious patient when performed with a rigid bronchoscope. In the intubated patient the endotracheal tube must be removed before and replaced after the bronchoscopy.

The advent of the flexible fibreoptic bronchoscope has

\section{Summary}

Experience with therapeutic bronchoscopy using the fibreoptic bronchoscope in intensive care has shown it to be a useful procedure. The Nosworthy connection has also been modified to allow intermittent positive-pressure ventilation to be maintained during bronchoscopy. This procedure is valuable in those cases where sputum or blood are retained in the airways despite adequate physiotherapy and endotracheal suction. Fibreoptic bronchoscopy should be available as a routine service in intensive therapy units.

\section{Introduction}

Despite advances in intensive care practice the problem of atelectasis due to retained sputum or blood clot is occasionally encountered. In patients breathing spontaneously coughing may be inadequate after surgery or chest wall trauma and may result in sputum retention despite dedicated physiotherapy. Patients who are intubated may also retain sputum because it is not always possible to pass the suction catheter into the left main bronchus, and the upper lobe bronchi are never accessible to blind suction.

In these circumstances therapeutic bronchoscopy is indicated to allow suction under direct vision. This procedure is seldom carried out in many units, however, partly because it can be

\footnotetext{
Division of Anaesthesia, Clinical Research Centre, Northwick Park Hospital, Harrow, Middlesex HA1 3UJ

J S MILLEDGE, MD, FRCP, consultant physician and MRC scientific staff member
}

changed the position. The fibreoptic scope can be passed through the endotracheal tube with continued intermittent positive-pressure ventilation in patients on ventilators, or in patients breathing spontaneously it can be passed through the nose under local anaesthetic with very little discomfort to the patient. The whole bronchial tree to segmental level including the upper lobes can be suctioned under direct vision.

Lindholm et $a l^{1}$ from Sweden and O'Donnell ${ }^{2}$ from Australia have written on the use of fibreoptic bronchoscopes in intensive care, and Sackner ${ }^{3}$ from the USA mentions this use in his review, but I am not aware of any reports of experience in this country.

\section{Apparatus}

Most of our experience has been gained with the Olympus BF-5B2 scope, which we keep on a trolley with its light source and instruments in the endoscopy room. From there it can be quickly taken to the patient's bedside in the ward or intensive therapy unit.

So that we can continue intermittent positive-pressure ventilation when indicated during the procedure a standard Nosworthy connector was modified by adding a side arm to the outside of the rightangle curve (fig 1 ). This side arm carries an $O$ ring which gives a near airtight seal around the bronchoscope while allowing easy movement in and out of the endotracheal tube. Modifications for Cobb's connectors for the same purpose have been described. ${ }^{45}$ The fibreoptic bronchoscope is also shown in fig 1 , and a diagram of its end, showing the layout and dimensions of the suction channel, light bundles, and optics channel is shown in fig 2.

\section{Technique}

In patients not on ventilators the usual technique for diagnostic bronchoscopy is used. Premedication is usually with lorazepam 


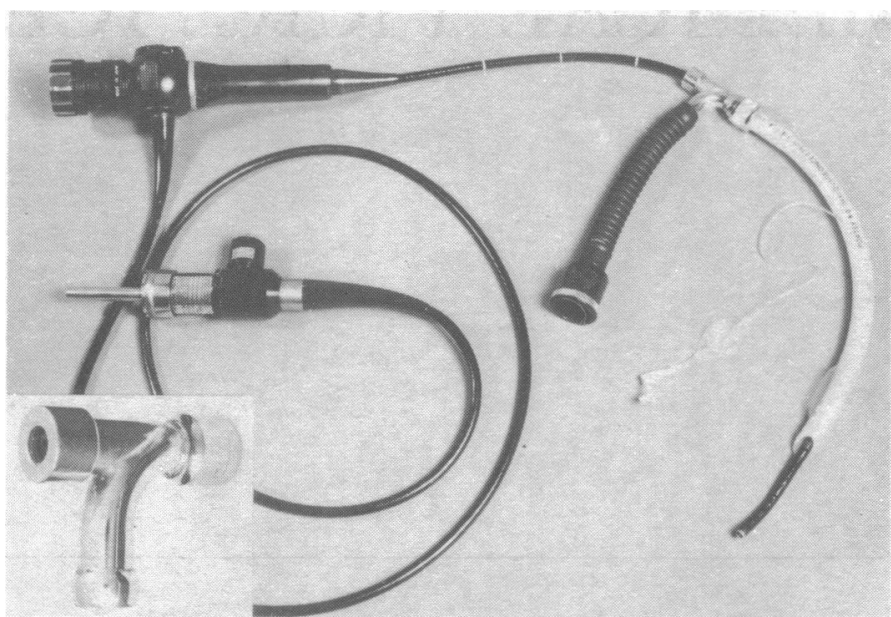

FIG 1-Olympus BF-5B2 fibreoptic bronchoscope passed through $9 \cdot 0-\mathrm{mm}$ endotracheal tube and modified Nosworthy connector. Detail of connector is shown inset.

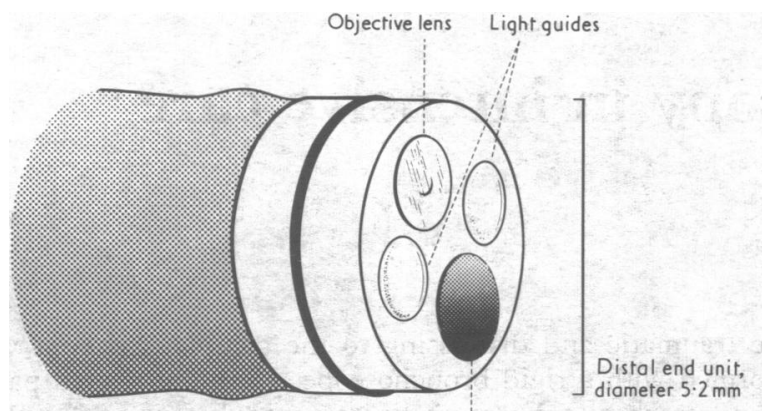

Suction channel, infernal diameter $1.9 \mathrm{~mm}$

FIG 2-Distal end of bronchoscope, showing dimensions and layout of light bundles, optical bundle, and suction channel.

$2.5 \mathrm{mg}$. The nostrils and pharynx are sprayed with lignocaine aerosol. The scope is lubricated with lignocaine jelly and introduced via the nostrils until the cords are visualised. These are anaesthetised with $2 \mathrm{ml}$ of $4 \%$ lignocaine through the scope. And $2 \mathrm{ml}$ of $2 \%$ lignocaine is delivered to the trachea, carina, and both main bronchi.

Patients not on intermittent positive-pressure ventilation undergo normal diagnostic bronchoscopy. Premedication is usually with lorazepam $2.5 \mathrm{mg}$. The nostrils and pharynx are sprayed with lignocaine aerosol. The scope is lubricated with lignocaine jelly and introduced via the nostrils until the cords are visualised. These are anaesthetised with $2 \mathrm{ml}$ of $4 \%$ lignocaine through the scope. And $2 \mathrm{ml}$ of $2 \%$ lignocaine is delivered to the trachea, carina, and both main bronchi.

In patients on intermittent positive pressure ventilation the scope is introduced via the endotracheal tube using some form of adaptor to allow ventilation to be continued. Bronchoscopy can be carried out under local anaesthetic, or a general anaesthetic can easily be started. An important limitation of this route is that the minimum size of endotracheal tube that allows passage of the scope and adequate ventilation around it is $8.5 \mathrm{~mm}$, and $9 \mathrm{~mm}$ is preferable. Fibreoptic bronchoscopy does interfere with air flow, resulting in some reduction in $\mathrm{PaO}_{2}{ }^{6} \mathrm{It}$ is therefore advisable to increase the amount of oxygen in the inspired air to patients with any tendency to hypoxia. In spontaneously breathing patients this can be done by using any disposable mask with a hole cut to take the scope.

Introducing the scope is not difficult, but even experienced bronchoscopists have some difficulty in finding their way around the bronchial tree on changing from the rigid to the flexible scope. The direction of the scope, of course, now gives no clue about what is being observed and the orientation will be inverted if the scope is made to take a $90-180^{\circ}$ turn in the nose and pharynx. Also, the optics are such that a small bronchus close to the tip of the scope appears to have the same diameter as a large bronchus slightly further away. The presence of mucus or blood obscuring the field at intervals adds to the problem.
A detailed knowledge of bronchial anatomy is therefore required, and this comes only with experience. This is best gained in the rather easier conditions of diagnostic bronchoscopy before embarking on therapeutic procedures.

Secretions and blood are suctioned under direct vision from the entire bronchial tree down to at least segmental level. Viscid secretions and blood clot will have to be loosened with a 10-ml bolus of saline. The tip of the scope is plunged into the secretions with suction applied and the scope is withdrawn. This pulls out a column of mucus, which eventually thins to a critical diameter, allowing it to pass rapidly up the 2-mm suction channel. The process is repeated, if necessary with more saline, until the visible tree is cleared. We have yet to encounter secretions which could not eventually be removed in this way. Further clearing may be achieved by instilling saline in time with the respirator during inflation so that saline is carried out to peripheral airways and returned during expiration, together with mucus plugs.

\section{Clinical experience and comment}

In the past year 21 therapeutic bronchoscopies have been carried out on 17 patients. Two were performed on the ward and the remainder in the intensive therapy unit. Ten bronchoscopies were carried out with intermittent positive-pressure ventilation via an endotracheal tube, the indication being retained sputum in all except one patient, who had inhaled stomach contents after cardiac arrest. Eleven bronchoscopies
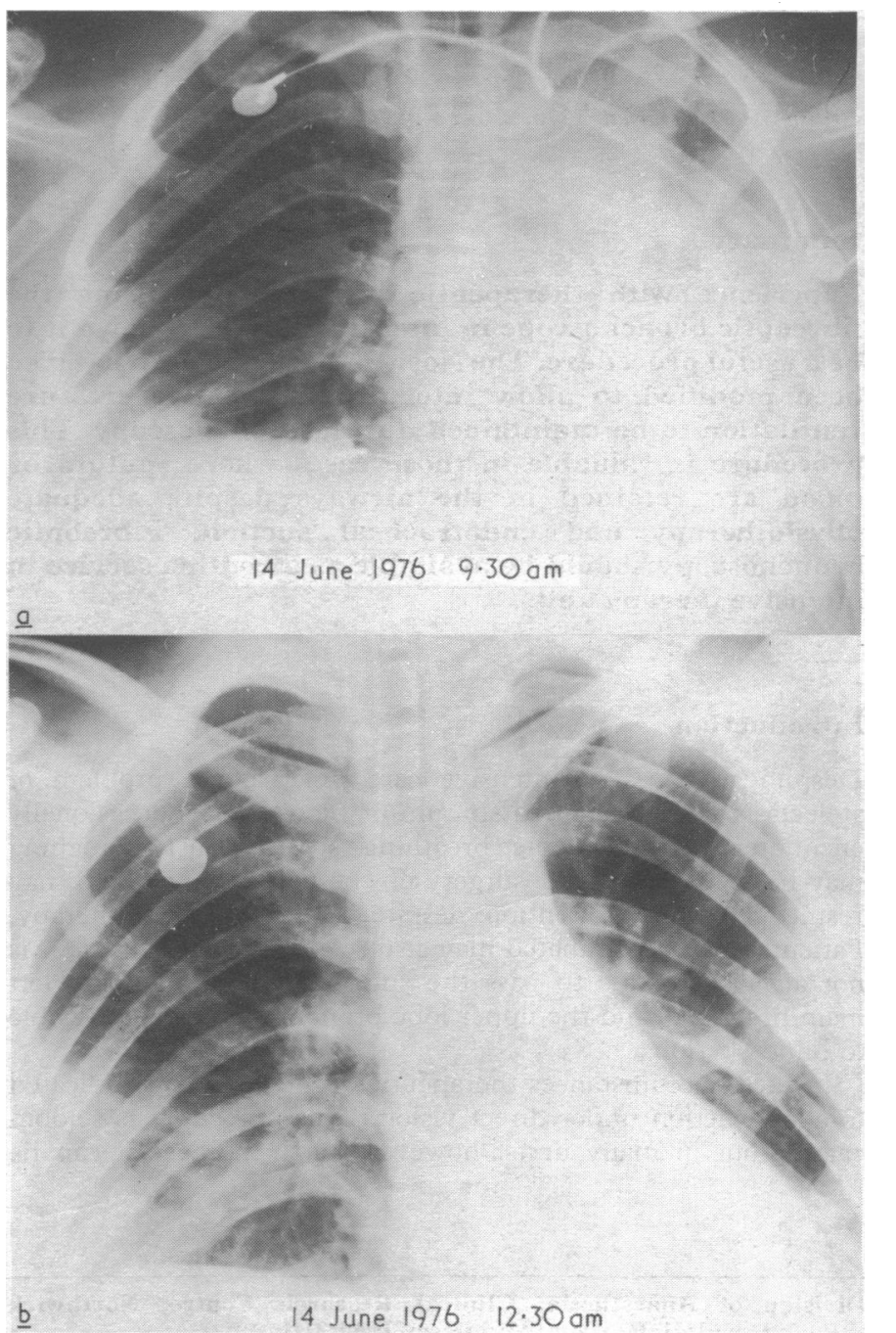

FIG 3-Chest $x$-ray picture of 17 -year-old youth injured in a traffic accident before $(a)$ and after $(b)$ bronchoscopic aspiration of blood clot in left bronchial tree. 
were performed on spontaneously breathing patients via the nasal route under local anaesthesia. The indication was usually for retained sputum with lobar collapse. Bronchoscopy improved the air entry in all cases, quite dramatically so in some. One such case is illustrated in fig. 3.

This 17-year-old youth sustained contusion of his right lung and a pneumothorax due to a motor cycle accident. The pheumothorax was successfully treated by an intercostal drain, but over the next three days, despite physiotherapy, first his left lower lobe and then his left upper lobe collapsed (fig $3 a$ ). This collapse was resistant to hourly physiotherapy. Fibreoptic bronchoscopy showed some increased secretions in the right tree and complete occlusion of the left main bronchus by blood clot and mucus. This was aspirated and was seen to extend to the segmental orifices of both lobes. Eventually all visible blood was aspirated. The left chest movement and air entry were dramatically improved, as was the chest x-ray picture (fig $3 b$ ).

There have been no complications from the procedure and it is well tolerated, even by very sick patients. Therapeutic bronchoscopy with a flexible fibrescope is therefore a valuable procedure in selected cases and should be available in intensive therapy units.

\section{References}

${ }^{1}$ Lindholm, C E, Critical Care Medicine, 1974, 2, 250.

2 O'Donnell, J E, Anaesthesia and Intensive Care, 1975, 3, 139.

3 Sackner, M, American Review of Respiratory Disease, 1975, 111, 62.

4 Lett, Z, and Ong, G B, Anaesthesia, 1974, 29, 623.

5 Lewis, R B, Anaesthesia, 1974, 29, 625.

6 Albertini, R E, et al, Fournal of the American Medical Association, 1974, 230, 1666.

\title{
Bone and Foint Diseases
}

\section{Hip fractures up to date}

\author{
P A RING
}

\section{RADIOGRAPHS}

Good anteroposterior and lateral films are necessary not only

Fractures of the femoral neck are the commonest serious leg injuries seen in the elderly. They have a high associated morbidity and mortality, not because of the injury but mainly because of the physical and mental consequences in the elderly of staying in hospital with a period of relative immobility. Both morbidity and mortality can be diminished by early and energetic treatment, and most of these patients may, with a combination of good orthopaedic and geriatric care, return to their previous environment with little or no additional disability.

Although a history of a fall in these patients is common, some will admit that the leg just gave way, and the injury is clearly associated with a senile osteoporosis. Thus many of these fractures are caused by disease.

\section{Diagnosis}

The history of a fall, or the story that the leg suddenly gave way, is a characteristic one, and the diagnosis in most elderly patients should not be in doubt. The leg lies in an externally rotated position and appears shortened, and any attempt at movement is painful. In some patients, however, the fracture may be impacted, and indeed walking is sometimes possible although uncomfortable after such an injury. The risk of neglect in these patients is obvious; such a fracture, which is a relatively favourable one, may disengage and become displaced.

The immediate management is the provision of suitable analgesics. Splinting the limb by tying it to the opposite member is a useful way of diminishing the discomfort during a journey to hospital. lesion since this influences the orthopaedic management to a considerable extent. Three main groups of fracture can be identified.

\section{Subcapital}

The fracture line is essentially at the junction of head and neck. It may lie in a relatively horizontal plane or may incline more to the vertical; the latter position is more unfavourable since the fracture is inherently unstable. Subcapital fractures may be classified again in more than one group.

Impacted-There is no disengagement at the fracture site; indeed, the outer side of the fracture is impacted into the femoral neck giving an essentially stable position. These are the fractures on which walking is often possible after the injury has occurred.

Undisplaced-These are very similar to the impacted fractures. There is no appreciable displacement on the femoral head relative to the neck, but both parts of the femur are disengaged at the fracture line.

Displaced-Here the amount of displacement may vary widely from a simple varus angulation of the neck to a complete separation of neck and shaft.

\section{Basal neck fractures}

Basal neck fractures occur at the junction of the neck and the trochanteric region; separation of the fragments may vary from slight to a very major angulation.

\section{Trochanteric}

The fracture line passes through the trochanteric region separating the lesser trochanter that lies as a free fragment and occasionally the greater trochanter as well. Displacement may again vary in severity 